\title{
IDENTIFICACIÓN DE CADMIO Y PLOMO EN LOS CULTIVOS DE CACAO UBICADOS EN LA ZONA DE SATIPO - JUNÍN
}

\author{
IDENTIFICATION OF CADMIUM AND LEAD IN COCOA CROPS \\ LOCATED IN THE SATIPO - JUNIN ZONE
Jimmy Rosales-Huamaní ${ }^{*}{ }^{\star}$, Luis Centeno-Rojas ${ }^{10}$, Jhomara Cajacuri-Perez ${ }^{3(0)}$

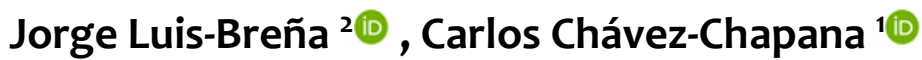

\author{
'Universidad Nacional de Ingeniería, Facultad de Ingeniería Geológica, Minera y Metalúrgica, Lima, Perú \\ ${ }^{2}$ Universidad Nacional de Ingeniería, Facultad de Ingeniería Química y Textil, Lima, Perú \\ 3 Universidad Nacional Mayor de San Marcos, Facultad de Ingeniería Química , Lima, Perú
}

Recibido (Received): 16/11/2020 Aceptado (Accepted): 30/06 / 2021

\begin{abstract}
RESUME
Actualmente existe un grave problema en los cultivos de cacao de las diversas regiones del Perú debido a la presencia de metales pesados en dichos cultivos. Las altas concentraciones de metales pesados en cultivos de cacao prohíben su exportación pues la Comunidad Europea ha establecido normativas alimentarias indicando los niveles máximos permisibles de metales pesados y demás elementos en alimentos. Se consideran que las posibles fuentes de contaminación del Cd en los cultivos de cacao pueden provenir de actividades naturales como actividades volcánicas, procesos de erosión y sedimentación en rocas que contiene minerales con un alto contenido en estos metales pesados. También esta contaminación es debido a las actividades antropogénicas tales como los yacimientos asociados a las minas, la agricultura (uso de fertilizantes), al procesamiento de los minerales y el uso indiscriminado de combustibles fósiles. Debido a que actualmente, no se tiene información de contenidos de metales pesados como Cadmio ( $\mathrm{Cd}$ ) y Plomo $(\mathrm{Pb})$ en una zona de estudio ubicada en Satipo-Junín, en el presente trabajo hacemos un estudio de dicha región, utilizando una metodología para la obtención de las concentraciones de metales de $\mathrm{Cd}$ y Pb presentes en el suelo de cultivos de cacao tipo forastero. Como consecuencia de este estudio determinamos y concluimos que las concentraciones obtenidas de $\mathrm{Cd}$ y $\mathrm{Pb}$ en suelos son menores a los estándares establecidos por el Ministerio de Medio Ambiente (MINAM), además generamos el mapa geoquímico de la zona de estudio con estos metales.
\end{abstract}

Palabras Clave: contaminación de suelos, metales pesados, cadmio, plomo

\section{ABSTRACT}

There is now a serious problem in cocoa crops in various regions of Peru due to the presence of heavy metals in these crops. The high concentrations of heavy metals in cocoa crops prohibit their export because the European Community has established food regulations indicating the maximum permissible levels of heavy metals and other elements in food. It is considered that the possible reasons for the contamination of the $\mathrm{Cd}$ in cocoa crops may come from natural activities such as volcanic activities, erosion processes and sedimentation in rocks that contain minerals with a high content of these heavy metals. This contamination is also produced by anthropogenic activities such as mining of associated deposits, agriculture (use of fertilizers), smelting of minerals and burning of fossil fuels. Due to the fact that there is currently no information on the content levels of heavy metals as Cadmium ( $\mathrm{Cd}$ ) and Lead $(\mathrm{Pb})$ in a study area located in Satipo-Junín. In this work, we study this region, using a methodology to obtain the concentrations of $\mathrm{Cd}$ and $\mathrm{Pb}$ metals present in the soil of foreign-type cocoa crops. As a consequence of this study we determined and concluded that the concentrations obtained of $\mathrm{Cd}$ and $\mathrm{Pb}$ in soils are inferior to the standards established by the Ministry of Environment (MINAM), in addition we generated the geochemical map of the study zone with these metals.

Keywords: soil contamination, heavy metals, cadmium, lead

\section{INTRODUCCIÓN}

* Corresponding author.:

E-mail: jrosales@uni.edu.pe
En el Perú actualmente el cacao constituye un rubro de importancia en las exportaciones, generando empleo a muchas familias de productores y a toda la 
cadena de valor de ese producto. Sin embargo, las exportaciones del cacao hacia los mercados europeos se ven amenazadas por indicios de contaminación con metales pesados en el cacao exportable, debido a los niveles superiores hallados en comparación con los valores establecidos por la comunidad europea. Por lo tanto, la contaminación por metales pesados en alimentos se ha convertido en una preocupación en muchos países.

El Cd es un metal traza sin funciones biológicas esenciales, por el contrario, es tóxico para plantas, animales y humanos en bajas concentraciones. Por su toxicidad variada en diversos órganos de los seres humanos está clasificado como un carcinógeno humano por la Agencia Internacional de la Organización Mundial de la Salud para la investigación sobre el cáncer [2]. Parte del Cd se libera naturalmente en los suelos a través de la meteorización de las rocas, que típicamente contienen concentraciones, los altos insumos provenientes de fuentes antropogénicas como la minería, la fundición, la industria microelectrónica y los fertilizantes de roca han aumentado significativamente el contenido de cadmio en muchos suelos en todo el mundo [16].

$\mathrm{El} \mathrm{Pb}$ es un metal pesado que afecta diversos sistemas del cuerpo como son: nervioso, gastrointestinal, hematológico, cardiovascular y renal. Dado que el $\mathrm{Cd}$ y $\mathrm{Pb}$ se encuentran naturalmente en la corteza terrestre en forma de minerales, las plantas pueden absorberlo y acumularse en los cultivos agrícolas. Por lo tanto, pueden ingresar a la cadena alimentaria humana creando un riesgo potencial para la salud $[13,18]$.

Actualmente la exportación del cacao en el Perú, es un rubro importante que genera diversidad de empleos a muchas familias peruanas. Sin embargo, estas exportaciones de cacao a los mercados europeos se ven amenazadas por indicios de contaminación con metales pesados en el cacao destinado a la exportación. En consecuencia, debe evitarse la producción de cacao en suelos muy contaminados. A partir de ello realizaremos el muestreo de suelos de cultivos de cacao tipo forastero en la zona de estudio ubicada en Satipo-Junin, luego determinaremos las concentraciones de metales pesados como el $\mathrm{Cd}$ y $\mathrm{Pb}$. De esa manera podremos determinar si la zona de estudio presenta elevada concentración de dichos metales.

\section{ANTECEDENTES}

La investigación se realizará a partir de artículos relacionados a los metales pesados y su relación con el cacao. La búsqueda bibliográfica fue realizada en diferentes bases electrónicas a partir de trabajos realizados en los últimos 5 años.

DOI: https://doi.org/ 10.21754/tecnia.v21i2.1062
Luego las investigaciones de Takrama [18], mencionaron que el $\mathrm{Cd}$ es un contaminante medioambiental tóxico que se produce naturalmente y puede acumularse en los cultivos agrícolas y por lo tanto entran en la cadena alimentaria humana. En dicho trabajo también mencionan que el $\mathrm{Cd}$ ha sido detectado en el chocolate y en otros derivados del cacao. Asimismo, en Amankwaah [3], realizaron un estudio de seis metales pesados de cuatro de las principales regiones productoras de cacao en Ghana. Dichos resultados mostraron el contenido de $\mathrm{Cd}$ en la semilla de cacao, para luego comparar con los estándares permitidos de máximas concentraciones totales permitidas de metales en el cacao y poder obtener algunas conclusiones. Por otro lado, en Chávez [8], realizaron un estudio en el sur del Ecuador acerca de la concentración del cadmio en plantas y suelos, para ello se recogieron muestras de suelo en diferentes plantaciones a diferentes profundidades, mientras que las muestras de las plantas se tomaron de árboles cercanos. El contenido de $\mathrm{Cd}$ en los granos de cacao se encontró por encima del nivel crítico, generando preocupación en el consumo de chocolate a base de cacao.

Luego en el trabajo de Gramlich [10], mencionaron que la captación de Cd por parte del cacao, ha llamado la atención, luego de que la Unión Europea decidió establecer valores tolerables para los productos del cacao. En su estudio analizan las concentraciones de Cd en hojas, cáscaras y semillas de cacao en cultivos ubicados en Bolivia. Además, evaluaron otros parámetros para poder identificar qué factores influyen en la absorción de Cd por los arboles de cacao.

En Gramlich [11], realizaron un estudio para identificar las fuentes de $\mathrm{Cd}$ en los suelos de las plantaciones de cacao en Honduras, de esa manera tratan de identificar los factores que permiten su acumulación en el cacao. En su trabajo analizan 55 fincas de cacao en Honduras analizando las concentraciones de Cd en las hojas, cascara, semilla y granos de cacao, además tratan de encontrar relaciones con el tipo de suelo y árbol. Independientemente Abt [1], analizaron el cacao en polvo, chocolate con leche y productos de semillas de cacao que fueron comprados al por menor en tiendas minoristas de los Estados Unidos, determinando sus concentraciones de $\mathrm{Cd}$ y $\mathrm{Pb}$ en los productos del cacao utilizando un espectrómetro de masas de acoplamiento inductivo.

Luego en Arguello [7], mencionaron que la reciente regulación del $\mathrm{Cd}$ en el chocolate amenaza la sostenibilidad de la producción de cacao en el suroeste de América. A raíz de ello, en Ecuador, realizaron un trabajo a nivel nacional para identificar la distribución espacial de Cd en los granos de cacao, así como los factores del suelo y agronómicos involucrados.

Revista TECNIA Vol.31 N² Enero-Junio 2021 
En el trabajo de Meter [14], mencionaron que una mayor capacidad de intercambio catiónico involucra una mayor área superficial de las partículas que constituyen los suelos, lo que puede conducir a una disminución significativa de la biodisponibilidad del cadmio. Finalmente, en el trabajo de Anyimah [4], resumieron las últimas publicaciones relevantes disponibles sobre el $\mathrm{As}, \mathrm{Cd}, \mathrm{Pb}$ y $\mathrm{Hg}$ en granos de cacao y alimentos en base a cacao, con el fin de resaltar las brechas para alentar la investigación en esos temas. Los autores, concluyen que se requieren más investigaciones para aclarar el efecto de estos contaminantes en alimentos a base de cacao.

En nuestro país, existen diversos trabajos relacionados al tema del Cd. En Huamani [12], se hace referencia a que la incidencia de $\mathrm{Cd}$ y $\mathrm{Pb}$ en los suelos de los cultivos es un problema complejo de abordar para los stakeholders de las regiones cafetaleras y de cacao de Huánuco y Ucayali, lo que dificulta la agroexportación de estos productos. El autor evaluó concentraciones de $\mathrm{Cd}$ y $\mathrm{Pb}$ en muestras obtenidas de 22 parcelas suelos y hojas de plantaciones de cacao orgánico de la zona, encontrando niveles significativos de esos metales pesados. De la misma manera, Arévalo [6], sostienen que la demanda del cacao aumentó de modo significativo en los últimos años y que las regulaciones internacionales respecto a la presencia de $\mathrm{Cd}$ y $\mathrm{Pb}$ cada vez son más exigentes con relación a disminuir sus contenidos.

Diversos estudios en el Perú, han confirmado la presencia de trazas de $\mathrm{Cd}$, potencialmente tóxico. Lo que ha obligado al Estado peruano a promulgar la Resolución Ministerial, $N^{\circ}$ 0449-2018-MINAGRI [14], donde se encuentra la denominada Agenda de impacto rápido vinculada a los niveles máximos de $\mathrm{Cd}$ en productos específicos como el cacao y chocolate.

En el presente trabajo, al no tener información de metales pesados en la zona ubicada en Satipo-Junin, nos vemos en la necesidad de realizar el análisis de suelo en los sembríos de cacao forastero, para determinar el contenido de $\mathrm{Cd}$ y $\mathrm{Pb}$. Para poder realizar este análisis, se planteó recolectar muestras de la zona de estudio, y una vez obtenidas se enviaron al laboratorio de la FIGMM-UNI ubicada en la ciudad de Lima para el análisis químico por espectrometría de absorción atómica, donde se obtienen las concentraciones de estos metales en suelos. A partir de esta información se generó el mapa geoquímico de $\mathrm{Cd}$ y $\mathrm{Pb}$ de la zona de, siendo uno de los aportes más importante del estudio.

\section{METODOLOGÍA}

A partir de los trabajos mencionados anteriormente, se puede deducir que actualmente los productores de la zona de estudio no conocen la verdadera dimensión de la problemática del cacao y no tienen la capacidad para enfrentarlo. La metodología para lograr el aporte de nuestro trabajo se menciona a continuación:

\subsection{RECONOCIMIENTO Y UBICACIÓN DE LA ZONA DE ESTUDIO}

Geográficamente el área del proyecto de estudio se encuentra situada en el Centro poblado de Alto Cushiviani, distrito de Rio Grande, Provincia de Satipo, departamento de Junín. Se encuentra aproximadamente a $416 \mathrm{~km}$. al este de la ciudad de Lima además cuenta con un clima tropical y una temperatura que varía entre $16^{\circ} \mathrm{C}$ a $28^{\circ} \mathrm{C}$, las zonas de vida entre las que se encuentra el centro el poblado son bosque pluvial-premontano tropical y bosque húmedopremontano tropical. Para acceder a la zona del proyecto de estudio se toma la Carretera Central con la ruta La Oroya-Merced-Pichanaki, por ultimo tomar la ruta hacia Río Negro. El recorrido dura 12 horas aproximadamente.

El estudio se realizó en suelos arcillosos con un potencial de hidrógeno ligeramente ácido localizadas en el centro poblado de Alto Cushiviani. Las muestras recolectadas se enumeran y se les genera las siguientes coordenadas en UTM WGS84 como se muestra a continuación en la Tabla 1:

TABLA I

Información de coordenadas de las muestras monitoreadas según coordenadas WGS84

\begin{tabular}{cll}
\hline $\begin{array}{l}\text { PUNTOS DE } \\
\text { MUESTREO }\end{array}$ & NORTE & ESTE \\
\hline $\mathbf{1}$ & 8762180 & 532053 \\
$\mathbf{2}$ & 8762170 & 532077 \\
$\mathbf{3}$ & 8762160 & 532083 \\
$\mathbf{4}$ & 8762143 & 532093 \\
$\mathbf{5}$ & 8762182 & 532218 \\
$\mathbf{6}$ & 8762188 & 532242 \\
7 & 8762202 & 532196 \\
$\mathbf{8}$ & 8762226 & 532153 \\
\hline
\end{tabular}

En la Figura 1 se muestran las coordenadas de estudio y su ubicación en el mapa donde se tomaron las muestras. 


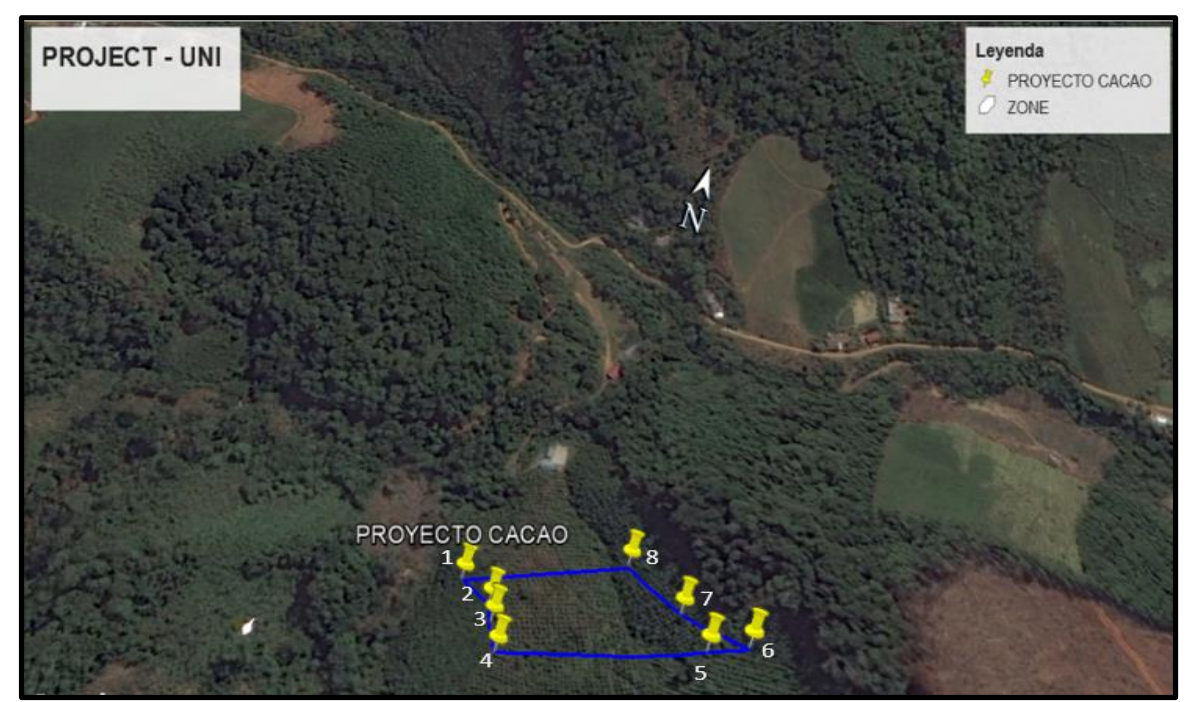

Fig. 1. Ubicación de coordenadas geográficas de los puntos de muestreo

\subsection{RECOLECCIÓN DE MUESTRAS}

Las muestras de suelo usadas para el estudio fueron recolectadas a profundidades de $30 \mathrm{~cm}$ como lo indica la guía de muestreo de suelos para el caso de suelo agrícola [17]. Se programaron muestras de $1 \mathrm{~kg}$ de cada una aproximadamente, las muestras fueron recolectadas con ayuda de una pala y homogenizadas dentro de un cubo plástico limpio para posteriormente ser almacenadas en bolsas estériles de polietileno las cuales fueron rotuladas con un marcador de tinta indeleble y enviadas al laboratorio de análisis de la FIGMM-UNI, donde fueron secadas al aire, molidas, pasadas por un tamiz y almacenadas a $22^{\circ} \mathrm{C}$.

La Figura 2 muestra las calicatas con una profundidad promedio de $30 \mathrm{~cm}$, excavadas para la toma de muestras de suelos.

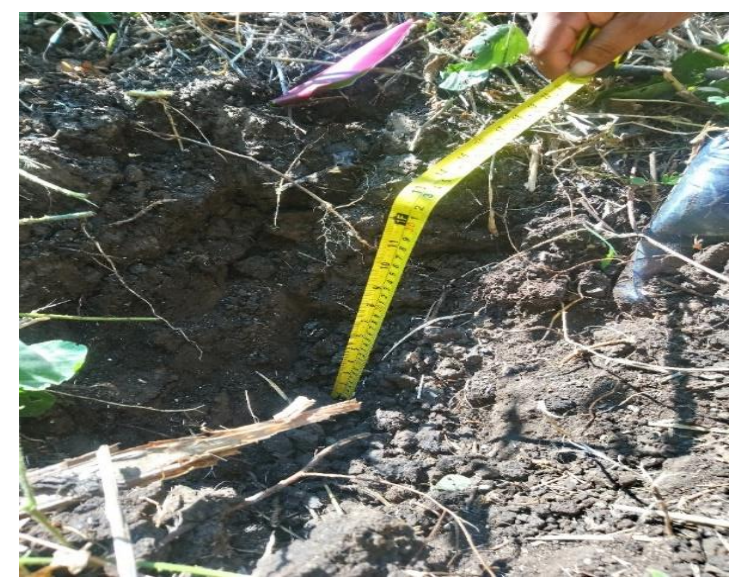

Fig. 2. Calicata de $30 \mathrm{~cm}$ de profundidad para la extracción de muestras

\subsection{PROCESAMIENTO DE MUESTRAS}

Con las muestras de suelo llegadas al laboratorio, se realizó primero la operación de secado de las muestras, que fue realizado en un horno de secado a una temperatura de $105{ }^{\circ} \mathrm{C}$ por 2 horas. Una vez seca la muestra, se realizó la operación de molienda, donde se utilizó un molino de jarras, ideal para trituración húmeda o seca. Este equipo proporciona una molienda homogénea, lo que permite llevar la muestra a una granulometría deseada de 75 micras.

\subsection{ANÁLISIS QUÍMICO DE LAS MUESTRAS}

Antes de realizar el análisis químico de las muestras obtenidas, se realizó la digestión de las muestras con ácidos y luego se prepararon soluciones de las muestras de suelos de sembríos de cacao tipo forastero, para lograr ello independientemente se realizó lo siguiente:

\subsubsection{DIGESTIÓN DE LAS MUESTRAS DE SUELO}

El contenido de metales pesados fue determinado según la Enviromental Protection Agency (EPA 3050B) [20]. La digestión se realizó en un calentador y en los matraces de Kjeldahl de $100 \mathrm{ml}$.

A $0.5 \mathrm{~g}$ de la muestra seca y molida de suelo se agregó $0.5 \mathrm{~mL}$ de agua destilada y $10 \mathrm{~mL}$ de ácido nítrico $\left(\mathrm{HNO}_{3}\right)$ al $70 \%$, se dejó reaccionar a $100{ }^{\circ} \mathrm{C}$ durante 2 horas en baño maría. Luego se retira la muestra contenida en el matraz y se deja enfriar durante 15 minutos. Finalmente se agrega $3 \mathrm{~mL}$ de peróxido de hidrógeno al $30 \%$ y se calienta a $100{ }^{\circ} \mathrm{C}$ durante 1 hora más en baño maría.

Concluida la digestión con peróxido de hidrogeno se retira las muestras de la plancha y se dejan enfriar a temperatura ambiente. Se filtra con papel de filtro Revista TECNIA Vol.31 N² Enero-Junio 2021 
whatman $\mathrm{N}^{\circ} 42$, en fiolas de $25 \mathrm{~mL}$, luego se lava con agua destilada hasta enrasar a un volumen de $25 \mathrm{~mL}$, se tapa y se agita [15].

\subsubsection{ANÁLISIS POR ABSORCIÓN ATÓMICA}

Las muestras en solución obtenidas del proceso de digestión fueron analizadas con ayuda de un Espectrofotómetro de Absorción Atómica (AAS) marca GBC. Scientific Equipment, modelo XplorAA. Se analizaron los metales pesados por triplicado. El elemento $\mathrm{Cd}$ a una longitud de onda de $228,8 \mathrm{~nm}$ y el $\mathrm{Pb}$ a una longitud de onda de $217 \mathrm{~nm}$.

\section{ANÁLISIS DE MUESTRAS}

Los resultados de las concentraciones obtenidas de $\mathrm{Cd}$ y $\mathrm{Pb}$ en suelos son mostrados en la Tabla 2.

TABLA II

Determinación de las concentraciones de $\mathrm{Cd}$ y $\mathrm{Pb}$ en las muestras de suelos mediante espectroscopia de absorción atómica

\begin{tabular}{ccc}
\hline MUESTRA & \multicolumn{2}{c}{$\mathrm{C}(\mathrm{mg} / \mathrm{kg})$} \\
& $\mathrm{Cd}$ & $\mathrm{Pb}$ \\
\hline 1 & 1.00 & 10.50 \\
2 & 1.50 & 30.50 \\
3 & 2.50 & 40.50 \\
4 & 1.00 & 6.000 \\
5 & 0.50 & 5.000 \\
6 & 1.00 & 20.50 \\
7 & 1.50 & 36.00 \\
8 & 1.00 & 6.500 \\
\hline
\end{tabular}

El análisis estadístico llevado a cabo con los datos muéstrales fue de comparación de medias por la t- student y análisis de correlación de Pearson entre las concentraciones de $\mathrm{Cd}$ y $\mathrm{Pb}$. La media de la concentración del Cd en suelos es 1,25 $\pm 0.598 \mathrm{mg} / \mathrm{kg}$ [9] y para el $\mathrm{Pb}$ es 19,44 $\pm 14,53 \mathrm{mg} / \mathrm{kg}$ [9]. De las pruebas de normalidad de los datos con relación al $\mathrm{Cd}$ y al $\mathrm{Pb}$ el estadístico de Ryan-Joiner (similar a Shapiro-Wilk) es igual a 0,958 para ambos elementos y en tanto que el $p$ valor $>0,1$ se acepta la hipótesis nula de que los datos tiene una distribución normal. Considerando que los estándares permisibles del MINAM establecen límites permisibles en suelos para Cd de $1.4 \mathrm{mg} / \mathrm{kg}$ y para $\mathrm{Pb}$ de $70 \mathrm{mg} / \mathrm{kg}$ en suelo agrícola, se aplicó la prueba T para evaluar si las medias presentan diferencias significativas con estos valores permisibles. En el caso del $\mathrm{Cd}$ el valor de $\mathrm{T}$ es $-9,84$, con un $\mathrm{p}$-valor $=0,000$, con lo concluimos que no existen diferencias significativas entre ambos valores, con lo que se puede concluir que el contenido de $\mathrm{Cd}$ en los suelos no supero los límites permisibles establecidos por el MINAM. Por otro lado, aplicando el t-student para el $\mathrm{Pb}$ se determinó que el valor $\mathrm{T}$ es igual a $-9,84$, con un p-valor de 0,000 , con la que se acepta la hipótesis alterna de que la media de contenido de $\mathrm{Pb}$ en los suelos es menor al límite permisible establecido por el MINAM.

Finalmente, se ha determinado que la correlación de Pearson entre ambos contenidos es $r=0,866$ una correlación positiva alta, con un $\mathrm{p}$-valor=0,005.

Con las mediciones tomadas en la región de estudio, se generaron los mapas geoquímicos de la distribución de concentración de $\mathrm{Cd}$ y $\mathrm{Pb}$ en los suelos utilizando el método de interpolación llamado kriging, para ello se utilizó el software ArcGIS [5].

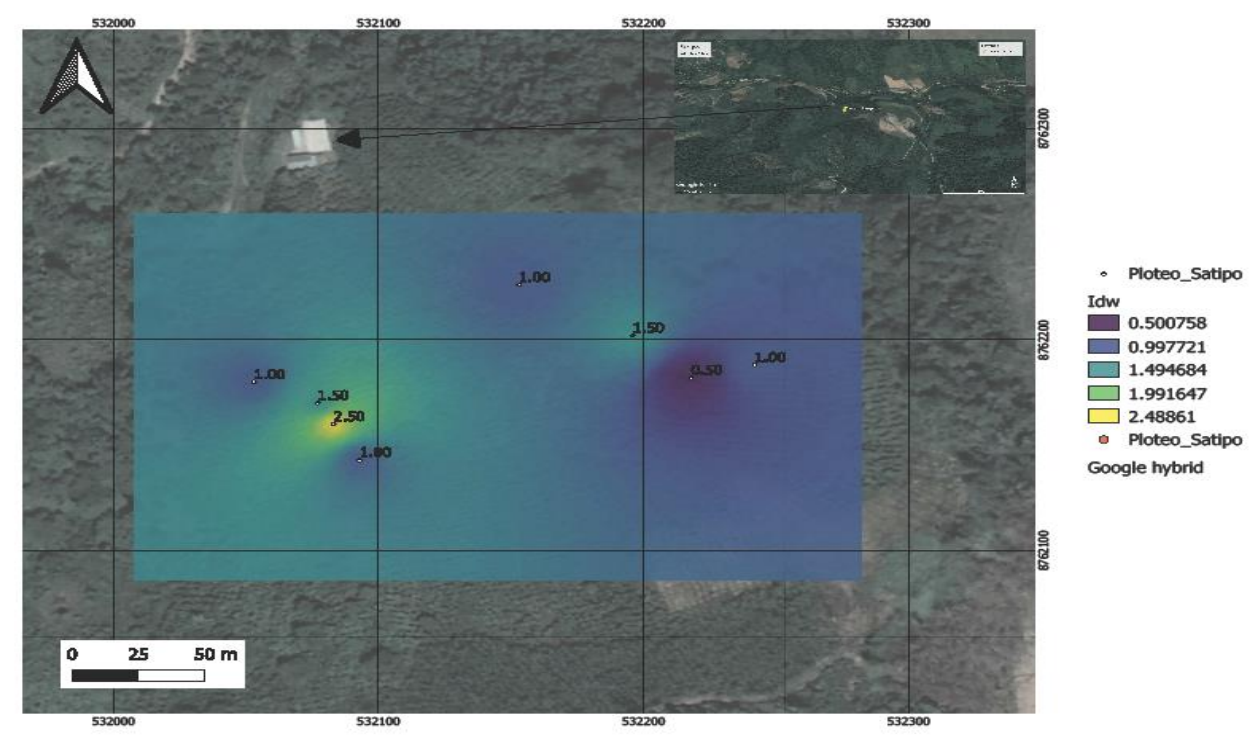

Fig. 3 Mapa geoquímico de Cd en la zona de estudio 


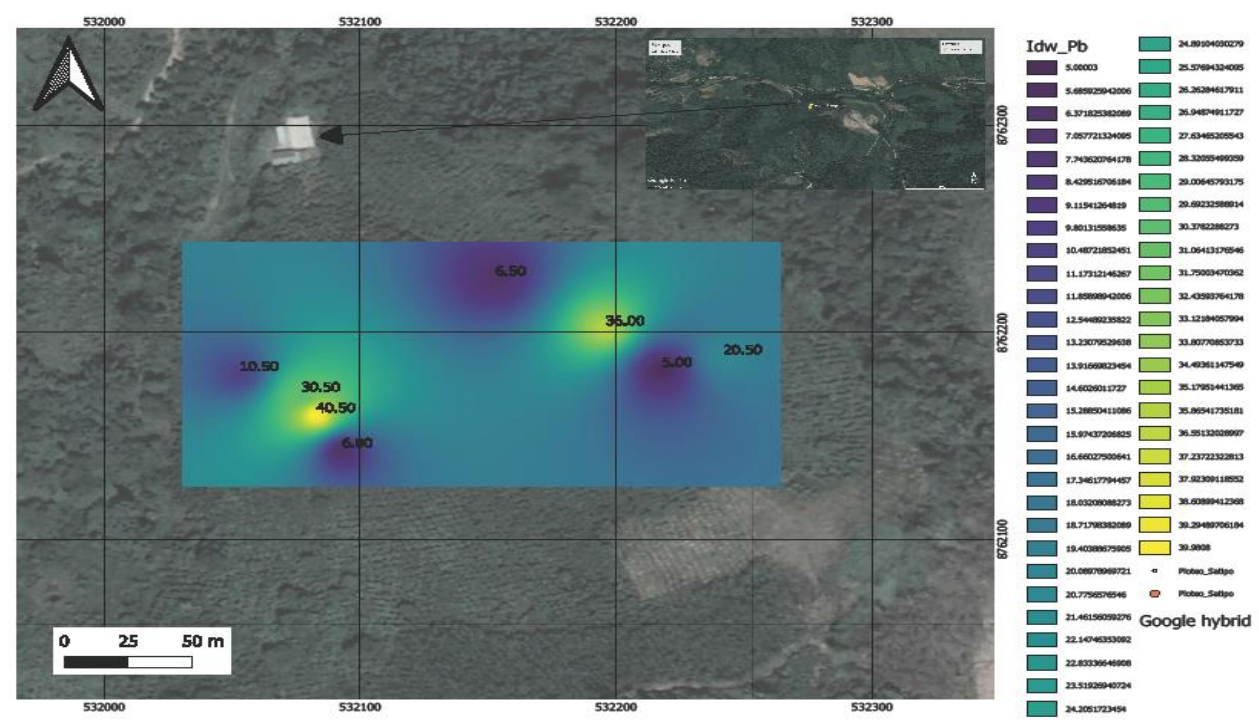

Fig. 4. Mapa geoquímico de $\mathrm{Pb}$ en la zona de estudio

\section{CONCLUSIONES}

- Los datos obtenidos en este estudio confirmaron que, para el caso de suelos, la media de concentración de $\mathrm{Cd}$ fue $1.25 \mathrm{mg} / \mathrm{kg}$ que se encuentra por debajo de los estándares permisibles del MINAM cuyo valor es $1.4 \mathrm{mg} / \mathrm{kg}$. En el caso del $\mathrm{Pb}$ la media de la concentración fue $19.44 \mathrm{mg} / \mathrm{kg}$ y también se encuentra por debajo de los estándares del MINAM.

- Si bien es cierto que el promedio de concentración $\mathrm{de} \mathrm{Cd}$ y $\mathrm{Pb}$ es menor a los estándares permitidos, es necesario constantemente estar monitoreando la zona de estudio y medir periódicamente diversas propiedades como $\mathrm{pH}$, conductividad eléctrica y concentración que están relacionadas con la movilidad del Cd, evitando la absorción del Cd por las plantaciones de cacao.

- Los mapas geoquímicos delimitan las zonas aptas para el cultivo del cacao, dado que los niveles de $\mathrm{Cd}$ y $\mathrm{Pb}$ se encuentran por debajo de los límites permisibles establecidos por el MINAM.

\section{AGRADECIMIENTOS}

Se agradece al Vicerrectorado de Investigación de la UNI por el financiamiento de la Investigación desarrollada.

\section{REFERENCIAS}

[1]E. Abt, J. Fong Sam, P. Gray,y L. P. Robin, "Cadmium and lead in cocoa powder and chocolate products in the US Market", Food Addit Contam Part B Surveill, vol. 11, no 2,pp. 92-102, jun. 2018.

[2]International Agency for Research on Cancer.(27, sep 2021). Agents Classified by the IARC Monographs, Volumes 1-129 [En línea]. Disponible en: https://monographs.iarc.fr/agents-classified-by-theiarcl

[3]D. Amankwaah, W. A. Nnuro, J. Awudza, y S. Afful, "Determination of heavy metals in cocoa beans from some major cocoa growing regions in Chana". Food Science and Technology, vol. 16, no 1, p. 225. 3, 2015

[4]E. Anyimah-Ackah, I. W. Ofosu, H. E. Lutterodt, y G. Darko, "Exposures and risks of arsenic, cadmium, lead, and mercury in cocoa beans and cocoa-based foods: a systematic review". Food Quality and Safety, vol. 3, no 1, pp. 1-8, 2019.

[5]ESRI, ArcGIS Pro. California, ESRI, 2020. [En línea]. Disponible en: https://www.esri.com/es-es/arcgis/products/arcgis-pro/overview

[6]E. Arévalo-Gardini, M. E. Obando-Cerpa, L. B. Zúñiga-Cernades, C. O. Arévalo-Hernández, V. Baligar y H. Zhenli, "Metales pesados en suelos de plantaciones de cacao (Theobroma cacao L.) en tres regiones del Perú”. Ecología Aplicada, vol. 15, no 2, pp. 81-89, sep. 2016.

[7]D. Argüello, E. Chavez, F. Lauryssen, R. Vanderschueren, E. Smolders, y D. Montalvo, "Soil properties and agronomic factors affecting cadmium concentrations in cacao beans: A nationwide survey in Ecuador". Science of the total environment, vol. 649, pp. 120127, 2019.

[8]E. Chávez, Z.L. He, P.J. Stoffella, R. S. Mylavarapu, Y.C. Li, B. Moyano y V. C. Baligar, "Concentration of cadmium in cacao beans and its relationship with soil cadmium in southern Ecuador". Science of the Total Environment, 533, pp. 205-214, 2015.

[9]Ministerio de ambiente. (s.f.). Decreto Supremo 002-2013[En línea]. Disponible en: https://fonamperu.org.pe/un-fondoambientalpara-el-peru/download/decreto-supremo-n-002-2013minam/

[10]A. Gramlich, S. Tandy, C. Andres, J.C. Paniagua, L. Armengot, M. Schneider y R. Schulin, "Cadmium uptake by cocoa trees in agroforestry and monoculture systems under conventional and organic", Science of The Total Environment, vol 580, pp. 677-686, feb. 2017 
[11]A. Gramlich, S. Tandy, C. Gauggel, M. López, D. Perla, V. Gonzalez, y R. Schulin, "Soil cadmium uptake by cocoa in Honduras". Science of The Total Environment, vol. 612, pp. 370-378, 2018.

Huamani-Yupanqui, H. A., Mansilla-Minaya, L. G., Florida-Rofner, N., \& [12]G. M. Neira-Trujillo, "Presencia de metales pesados en cultivo de cacao (Theobroma cacao L.) orgánico”, Acta agronómica, vol. 61, no 4, 2012.

[13]J. P. Méndez, C. A. G. Ramírez, A. D. R. Gutiérrez,y F. P. García, "Contaminación y fitotoxicidad en plantas por metales pesados provenientes de suelos y agua, Tropical and subtropical Agroecosystems, vol.10, no 1, pp. 29.44, 2009.

[14] A. Meter, R. Atkinson y B. LAbiberte, Cadmio en el cacao de América Latina y el Caribe : Análisis de la investigación y soluciones potenciales para la mitigación. Roma. Maccarese-Stazione, RM, Italia: Bioversity International, 2019.

[15]El Estado Peruano. (31, dic 2018). Resolucion Ministerial 449-2018$\mathrm{EF} / 10$ [En línea]. Disponible en: https://www.gob.pe/institucion/mef/normas-legales/235990-4492018-ef-10
[16]E. W. Rice, R. B. Baird, A. D. Eaton, y L. S. Clesceri. Standard methods for the examination of water and wastewater. Washington DC: American Public Health Association, p. 541, 2012.

[17]S. M. Ross. Sources and forms of potentially toxic metals in soilplant systems toxic metals in soil-plant systems, UK: Bistrol, pp. 3-25, 1994.

[18]M. Sabroso, A. Pastor. Guía sobre suelos Contaminados. CEPYME Aragon y Gobierno de Aragon: Zaragoza, España, 2004. [En línea]. Disponible

en:

https://www.academia.edu/29109561/Gu\%C3\%ADa_sobre_suelos_con taminados

[19]J. Takrama, A. A. Afrifa, K. Ofori-Frimpong, W. A. Jonfia-Essien,P. Agyemang y l. Galyuon, "Cadmium contamination of cocoa beans and cocoa growing agricultural soils of Ghana: There is no cause for public alarm", Peak Journal of Public Health and Management, pp. 56-61, 2015 [20]United States Environmental Protection Agency. Method 3050B: Acid Digestion of Sediments, Sludges, and Soils; Revision 2, U.S. EPA: Washington, DC, USA, 1996; vol. 2, pp. 1-12, 1996. 